\title{
Cylinders, planes, lines and points
}

\author{
Suggestions for a new conception of \\ the handshape parameter in sign languages
}

\author{
Onno Crasborn \\ Department of Linguistics, University of Nijmegen
}

\section{Introduction}

The phonological specification of lexical items in sign languages has centered around different properties of the whole hand. Its shape, orientation and location are specified for each sign, while changes in these properties result in movement. This paper contributes evidence for the claim that the phonologically specified articulator in sign languages is not always the hand as a whole. Rather, the articulator is argued to consist of a more abstract perceptual specification, which is typically articulated by the fingers. The evidence consists of the phonetic use of fingers that are flexed to different degrees for the purpose of articulating phonological specifications that do not refer to the fingers (i.e. are not articulations of handshape features). A set of perceptual categories is put forward which consists of zero-, one-, two- and three-dimensional objects, which can be seen as a first step away from the central role of the hand in all phonological descriptions and analyses since Stokoe (1960).

\section{The phonological specification of handshape}

Handshapes are traditionally characterized by specifying the selected fingers and their position. The selected fingers can for example be the index finger, the thumb, the pinky, the thumb and index, or all four fingers (excluding the thumb). The finger position can be extended, bent, or clawed (see images below, in which the selected finger is the index). ${ }^{1}$

Crasborn \& van der Kooij (2003) argue that the MCP joints of the fingers are not phonologically specified. Data to support the argument include the variation between 'straight' and 'bent' that was found, among other things, to correlate to specific combinations of location and orientation values. All positions between -15 


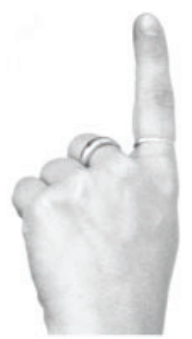

a. extended (no flexion)

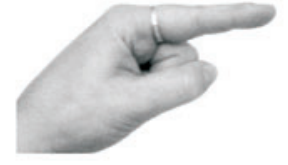

b. bent

(flexion at the 'metacarpophalangeal' or 'MCP' joint)

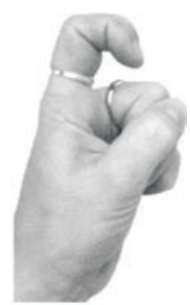

c. clawed

(flexion at the 'interphalangeal' or 'IP' joints)

\section{(1) Examples of finger position}

and 90 degrees flexion were found: it is not the case that there are two allophones (one with 0-degree flexion and one with 90-degree flexion) that appear in welldefined phonological contexts. The present paper further strengthens the argument against the specification of the MCP joints, by analyzing cases in which the MCP joints in signs with straight fingers can flex to different degrees. These will be presented and discussed in the next section.

A further property of all handshape models is that a particular specification for shape applies to all selected fingers; a complex exception concerns the shape specification [crossed], which refers to the 'crossing' of the index and middle fingers.

\section{Differential flexion of multiple selected fingers}

Signs with the finger configuration feature [extended] are typically realized as in Figure 2a: all specified fingers (all four in this example) are in exactly the same position. The three joints of each finger are all fully extended (0-degree flexion). However, the fingers can also be bent to different degrees at the most proximal (metacarpophalangeal or MCP) joint, which connects the fingers to the rest of the hand. Two examples of such a configuration are presented in Figure $2 b$, for four and two selected fingers, respectively.

Shapes like these have occasionally been discussed in the literature on American Sign Language (ASL). Baker-Shenk \& Cokely (1980) mention the rightmost handshape in (2) as a variant of the $\mathrm{V}$ shape. Brentari (1998) proposes a feature [stacked] to describe such an alternative form of V (as well as the highly similar K handshape from the fingerspelling alphabet), and proposes a redundancy rule to generate this alternative form in the context of the feature [spread]. The term 'stacked' is meant to describe the placement of one selected finger on top of the 


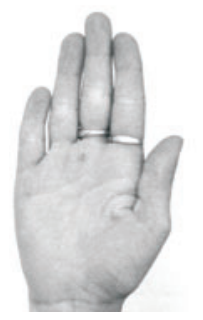

a. all fingers extended
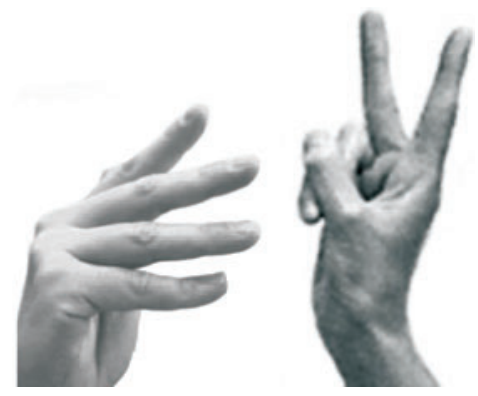

b. fingers flexed to different degrees

(2) Differential flexion of multiple selected fingers

next. In the middle handshape in (2), the index finger is stacked on top of the middle finger, the middle finger is stacked on top of the ring finger, and the ring finger is stacked on top of the pinky finger.

The claim is thus that in ASL the presence vs. absence of the feature [stacked] is an instance of allophonic variation: there are two clearly distinct surface forms and it depends on the phonological context which occurs when. In addition, [stacked] occurs in a few ASL signs with four selected fingers, such as FEw and MELT, in which the feature [spread] is absent and the fingers are bent at all joints but to different degrees; in such signs, the fingers are configured "roughly speaking, in the position needed to grip a racquet" (Brentari 1998:110). In a more descriptive phonetic approach, Johnson \& Liddell (2002) distinghuish two different stacked configurations: "radial stacking" and "ulnar stacking". In the former, the fingers on the ulnar (pinky) side of the hand are more flexed than the fingers on the radial (thumb) side; in the latter, the radial fingers are more flexed than the ulnar ones.

The radial form of stacking in which the pinky joints are flexed most and the index joints are flexed least also corresponds to many rest positions of the hand, as one can easily observe in daily life. This is related to the anatomical and physiological situation that there is an independent extensor muscle for the index finger and a joint flexor muscle for the middle, ring and pinky fingers (as noted by Ann 1993), but not the other way round, and secondly, the situation that the metacarpals (hand bones) of the ring and pinky finger are able to flex a little bit while the index and middle finger metacarpals cannot. Thus, it may well be easier to realize radial stacking than ulnar stacking, although this hypothesis would need further physiological corroberation.

In NGT, I have also observed the 'grip' handshapes mentioned by Brentari, in which all fingers are flexed to a certain degree at all joints. They occur in some realizations of the AS and $\mathrm{C}$ handshapes in signs such as TO-WASH (AS handshape) and vegetables ( $C$ handshape). However, differential finger flexion also occurs frequently in variants of signs with the finger configuration specification [extended], 
as illustrated in (2b). From impressionistic transcriptions, the degree of flexion of the different fingers seems to be rather variable. These phonetic variants have been found as steady-state configurations, as in NGT signs like SEE (two spread selected fingers) and WELCOME (four selected fingers), but the present investigation has also revealed many cases where the fingers flex at the MCP joints to different degrees during the sign. In signs such as TwENTY (two selected fingers) and oR (four selected fingers), the ulnar (thumb side) fingers flex most and the radial (pinky side) fingers flex least, giving rise to the configurations in the illustration above at the beginning or end of the sign.

Further examples of stacked configurations include PROOF, LICK, and DECEASED; 'stacking' movements also occur in ALREADY, MUCH, and WAVES. In each case, stacked variants have repeatedly been informally observed in multiple signers.

\section{A phonetic explanation for the observed variants}

I would like to propose three hypotheses to account for the variation in finger flexion. In the first place, the fact that the variation in flexion appears to be gradual rather than categorical suggests that these variants are not generated by a phonological rule generating allophonic variants (as suggested by Brentari 1998 for the alternation between $\mathrm{V}$ and $\mathrm{K}$ in ASL), but rather a matter of phonetic implementation (see Crasborn 2001 for a discussion of phonetic implementation in sign languages).

Secondly, I would like to propose that the 'stacked' forms are phonetic instances of specific phonological specifications for location and orientation - rather than resulting from handshape specifications alone. I hypothesize that these variants have the same phonological specification (namely extended selected fingers), while the differences in flexion at the MCP joints arise in the phonetic implementation component as a result of the aim to achieve an orientation target with relatively small articulatory actions of more proximal joints such as the wrist, forearm, and shoulder. This is illustrated in (3) for the sign welcome. In the citation form, two $B$ hands (all finges extended) with the palms pointing up move towards the body. In the variant illustrated here, the fingers of both hands still form a horizontal plane, but the right hand as a whole is not horizontal or 'palm up': the palm part of the right hand is pointing diagonally leftward-upward.

Thirdly, multiple selected fingers can be spread by abduction at the MCP joints (the middle finger typically stays fixed), but they can also be spread by flexion at the $\mathrm{MCP}$ joints alone, I hypothesize. Thus, the prototypical $\mathrm{V}$ and $\mathrm{K}$ handshapes would be completely identical in terms of handshape specification (two selected fingers, spreading), but the orientation of the plane constituted by the two fingers differs in the two cases if all other joints are in an identical position. In cases of different movement directions of the sign SEE (index and middle fingers spread, fingers 

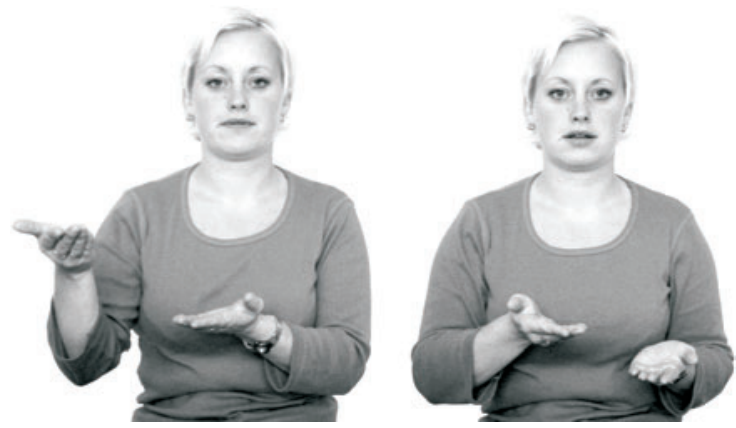

(3) Stacked fingers in weLCome (begin and end)

pointing upwards, back of the fingers pointing to the object location of the verb), the truly spread variant would be the easiest articulation in the citation form (1-SEE-2), while the stacked variant would be the easiest articulation in the form 1 -SEE-3 if the object location is to the right. This stacked form is 'easy' in the latter case because it reduces the amount of rotation of the forearm necessary for the correct orientation. In the citation form, on the other hand, stacking would require additional rotation of the forearm to achieve the correct orientation of the plane formed by the index and middle fingers; in this case spreading is the cheapest articulation.

The same is true of the other forms with stacked configurations, including the instance of WELCOME illustrated in (3). In the cases where the fingers flex to different degrees during the movement, the finger flexion limits the need for rotation of the forearm. In (4), this is illustrated for the palm orientation change in TWENTY.
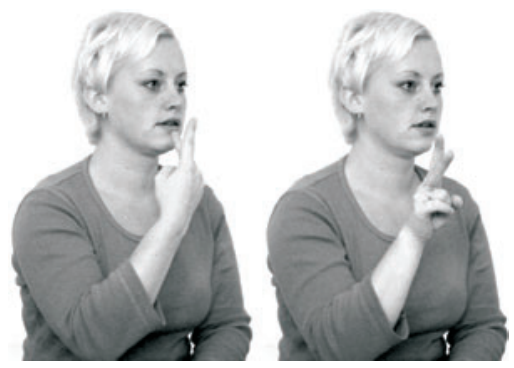

(4) Finger flexion as the articulation of a change in orientation in TWENTY

In the citation form, both index and middle finger are fully extended, fingers pointing upwards; the movement consists of a (near) 180-degree pronation of the forearm, changing the palm orientation from backward to forward. In the form illustrated in (4), the middle finger is flexed at the beginning of the sign while the 
index finger is flexed at the end of the sign; the rotation of the forearm is limited to about 100 degrees. If we consider the rotation of the triangular plane that the index and middle fingers outline, however, we see that the rotation is well over 100 degrees, approximating the 180 degree change in the citation form.

\section{Discussion: Implications for the phonological conception of the 'handshape' parameter}

The three hypotheses presented in the previous section to account for the articulatory variability in the realization of handshape targets in NGT suggest a new way of looking at what have traditionally been called 'handshapes'. If indeed the fingers adapt to realize orientation values in terms of the alignment of articulator planes to (abstract) location planes, as in the case of B and V handshapes illustrated above, would it not be possible to define the articulator in terms of such perceptual targets as well? We could then think of phonologically specified articulators of various dimensions, as outlined in (5). The same handshapes can be found as tokens of different perceptual targets: a B handshape can serve both as the articulation of a plane (in which case its flatness is emphasized) or of a block (in which case its threedimensionality is emphasized).

(5) A sketch of perceptual definitions of articulators of different dimensions

\begin{tabular}{llll}
\hline $\begin{array}{l}\text { no. of } \\
\text { dimensions }\end{array}$ & example articulator & $\begin{array}{l}\text { corresponding } \\
\text { handshape }\end{array}$ & NGT examples \\
\hline 0 & point & 1 & indexical signs \\
1 & line & 1 & FATHER, NORMAL \\
2 & plane & B & PLAY \\
& narrow plane & N & NEAT \\
& triangular plane & V & TWENTY, TWO \\
3 & block & B & car classifier \\
& cylinder & O & BAR \\
& open cylinder & C & COMMUNICATE \\
\hline
\end{tabular}

This is obviously a first sketch of an alternative view on handshapes, but it potentially offers solutions to a large set of problems of phonetic variability that have only recently received more attention (e.g. van der Kooij 2002). ${ }^{2}$ The abstract nature of these definitions would also allow for an easy explanation of the alternations in 'scale' in ASL discussed by Mandel (1981): sometimes variation occurs between one-handed and two-handed versions of signs, which appear to share a perceptual 
target. An example of such a target would be an imaginary cylinder, that can either be 'grasped' by an $\mathrm{O}$ hand (one-handed version), or by two $\mathrm{C}$ hands at some distance from each other. Such alternations appear to be more common in the productive lexicon and morphosyntax (classifier constructions) than in the frozen lexicon, but that does not imply that we do not need a phonological account for such alternations in form.

\section{Conclusion}

The present description of phonetic variation relating to the configuration of the fingers is clearly in its initial stages, but it forms an important step in the description of sign languages. Phonetic variation has not received much attention, and descriptions of phonetic variation that do occur typically limit themselves to the categories that are implicit in many of the transcription systems such as the KOMVA and HamNoSys notation systems (NSDSK 1988, Prillwitz et al. 1989).

The variation in finger configuration typically described as 'stacking' of fingers on top of one another was suggested to be the result of 'cheap' articulations of features relating to the orientation of the the articulator, which in turn is interpreted by looking at the location specification (Crasborn \& van der Kooij 1997, Brentari 1998).

I outlined a proposal to describe the active articulator in more abstract perceptual targets, rather than in terms of (relatively) concrete reference to individual fingers and their position with respect to the hand. For example, if there would be an articulator 'wide plane' in NGT, this perceptual target would most optimally be articulated by fully extending all fingers at all joints (and having the thumb parallel to the index finger in the same plane as the other fingers). Alternative articulations would be with 90 -degree MCP flexion at all joints, or different degrees of flexion at the different MCP joints. The concept of 'a plane formed by the fingers' that was proposed to characterize the 'stacked' handshapes found in NGT is a phonetic and phonological novelty, a new way of looking at signs. Such a plane forms a perceptual category that abstracts away from the articulation in terms of joint states.

This analysis can best be accommodated in a phonetic-phonological model that makes a clear distinction between perceptual categories (location, orientation, articulator shape/dimensionality) and articulatory categories (flexion and rotation of the joints of the arm and hand), such as the Functional Phonology model proposed by Boersma (1998) for spoken languages. Perceptual features constitute the lexical representation, while articulatory features needed for production are generated in the phonetic implementation process.

Future research will be aimed at investigating how useful this perceptual approach is for characterizing all handshapes. Also, the question needs to be answered how common the observed variation is in connected signing. Finally, it 
could be investigated whether the observed gradual variation in NGT occurs in other sign languages as well, or whether it is the case that languages like ASL only have the 'allophonic stacking' described in Johnson \& Liddell (2002) and Brentari (1998).

\section{Notes}

1. Other dimensions of handshape specification include spreading of more than one selected finger, and an 'aperture' relation between the opposed thumb and the selected fingers. These two dimensions do not directly impact the variant forms discussed in this paper, and will not be further discussed.

2. The above classification resembles to some extent the few earlier proposals for perceptual features to analyze ASL handshapes (e.g. Lane, Boyes Braem \& Bellugi 1976). Those proposals included a large set of distinctive features to characterize handshapes in terms of 'compact', 'broad', 'concave', etc. While these features correctly described the outcome of perceptual confusion studies and acquistion data, the proposals did not catch on because of their redundant nature.

\section{References}

Baker-Shenk, C. \& D. Cokely (1980) American Sign Language: a teacher's resource guide to grammar and culture. 2nd ed., 1991, T. J. Publishers, Silver Spring.

Boersma, P. (1998) Functional phonology. Formalizing the interactions between articulatory and perceptual drives. LOT, Utrecht.

Brentari, D. (1998) A prosodic model of sign language phonology. MIT Press, Cambridge MA.

Crasborn, O. (2001) Phonetic implementation of phonological categories in Sign Language of the Netherlands. LOT, Utrecht.

Crasborn, O. \& E. van der Kooij (1997) 'Relative orientation in sign language phonology'. In J. Coerts \& H. de Hoop, eds., Linguistics in the Netherlands 1997. John Benjamins, Amsterdam, 37-48.

Crasborn, O. \& E. van der Kooij (2003) 'Base joint configuation in Sign Language of the Netherlands'. In J. van de Weijer, V. van Heuven \& H. van der Hulst, eds., The phonological spectrum. Volume 1: segmental structure. John Benjamins, Amsterdam, 257-287.

Kooij, E. van der (2002) Phonological categories in Sign Language of the Netherlands. The role of phonetic implementation and iconicity. LOT, Utrecht.

Johnson, R. \& S. Liddell (2002) 'The phonological description of hand configuration'. Ms., Gallaudet University.

Lane, H., P. Boyes Braem \& U. Bellugi (1976) 'Preliminaries to a distinctive feature analysis of American Sign Language'. Cognitive Psychology 8, 263-289.

Mandel, M. (1981) 'Phonotactics and morphophonology in American Sign Language'. PhD dissertation, University of California at Berkeley.

NSDSK (1988) Notatie-systeem voor Nederlandse gebaren. Nederlandse Stichting voor het Dove en Slechthorende Kind, Amsterdam.

Prillwitz, S. et al. (1989) HamNoSys version 2.0. Hamburg notation system for sign languages: an introductory guide. Signum, Hamburg.

Stokoe, W. (1960) Sign language structure. 2nd ed., 1978. Linstok Press, Silver Spring, MD. 